\title{
ANALYSIS OF METHODS FOR RECONSTRUCTING PERIODICALLY MISSED SAR DATA ACQUIRED CLOSE TO NYQUIST
}

\author{
Muriel Pinheiro, Marc Rodriguez-Cassola, Pau Prats, Andreas Reigber \\ German Aerospace Center (DLR), Wessling, Germany \\ E-mail: muriel.pinheiro@dlr.de
}

\begin{abstract}
The cooperative nature of the TanDEM-X mission bistatic operation allows the periodically exchange of information between transmitter and receiver over the SAR acquisition. With the information gathered during the synchronization (sync) events it is possible to perform the phase and time referencing necessary for the calibration of the bistatic SAR image. However, given that transmitter and receiver are dedicated to the link during the sync events, the reception of SAR signal is periodically interrupted leading to gaps in the SAR raw data. Consequently, the processed SAR image will be corrupted, especially when acquiring close to the Nyquist rate. The paper addresses different approaches based on parametric and non-parametric spectral estimation to reconstruct the missing data and presents results with SAR data from TerraSAR-X and TanDEM-X.
\end{abstract}

Index Terms - TanDEM-X, bistatic, missing data, spectral estimation, reconstruction.

\section{INTRODUCTION}

Reconstruction of missing data is a common problem in signal processing. For SAR applications, the issue might be related with interrupted operation, new acquisitions modes with variation of the system PRF or with calibration and synchronization of bistatic spaceborne SAR, among others [1]. The missing information in the SAR raw data translates into corruption of the SAR image products, which can be more or less accentuated depending on the amount of missing information and on how close to Nyquist the system operates.

The problem of missing data is especially relevant for bistatic cooperative and non-cooperative SAR systems. For bistatic operation, missing data might occur due to uncorrelated errors on the transmitter and receiver antenna pointing vectors, which can lead to data loss at the positions corresponding to nulls of the antenna patterns. Furthermore, the lack of a common time referencing can cause miss-positioning of the receiver echo window and consequently data loss $[2,3,4]$. In cooperative bistatic systems, as is the case of TanDEM-X, there is a synchronization link between transmitter and receiver, a duplex periodic operation characterized by a certain frequency $f_{-}$syn much smaller than the system PRF. During each sync event a few synchronization and calibration pulses $n \_s y n$ are exchanged in order to allow time and phase referencing [2]. For the TanDEM-X mission, a trade-off between proper sampling of the oscillator noise and the minimization of aliasing (interpolator errors) leads to an $f \_s y n$ of
$5 \mathrm{~Hz}$. The number of exchanged pulses per event is usually of about 3 to 4 .

During each of the synchronization events no radar pulses are transmitted nor received. Due to interrupted reception, $n \_s y n$ range lines of raw data will be missed at the azimuthal times corresponding to the sync event. Furthermore, due to the interrupted transmission, every gap appears mirrored in the azimuthal signal at the position corresponding to the travelling distance of the not transmitted SAR pulses.

The TanDEM-X operational synchronization link frequency and number of exchange pulses per event and the related missing data are already enough to impair quality of the processed data, especially when large synthetic apertures are involved, as in the case of spotlight acquisitions. Therefore, in order to guarantee a minimum quality loss, reconstruction methods have to be applied [4]. If the system operates at low oversampling rates, as is usually the case for TanDEM-X acquisitions, the amount of data missed per sync period is larger than the allowed by the Nyquist criterion. Therefore, low order interpolators such as nearest neighbor, linear or cubic interpolators are not able to properly reconstruct the data, and more sophisticated algorithms must be considered.

This paper analyzes different approaches based on parametric and non-parametric spectral estimators to reconstruct missed SAR raw data. The viability of the use of such methods for reconstructing data from different scene characteristics is discussed and results using data from TerraSAR-X and TanDEM-X are presented.

\section{EFFECTS OF MISSING DATA IN SAR IMAGES}

The loss of raw data information at certain slow time instants leads to corruption of the data Doppler spectrum. For periodic data loss as the one caused by the sync link in bistatic cooperative systems, the resulting spectrum can be seen as the convolution of the original spectrum and the spectrum of a gap envelope assuming zero value at the positions correspondent to the sync events and at the related traveling time instants, and one everywhere else. The spectrum of such gap envelope is a combination of sinc functions whose side-lobes depend on the frequency and size of the missing information.

Considering the linearity of the SAR processing, every focused azimuthal line can also be seen as the $1 \mathrm{D}$ convolution of the original focused signal (processed without gaps) and a sinc envelope with high side-lobes. For a point target, for example, this will result in an increase of the side-lobes energy along azimuth. Therefore the point target azimuthal Impulse Response Function (IRF) will present increased maximum ambiguity power and increased Integrated Side-Lobe Ratio (ISLR). 


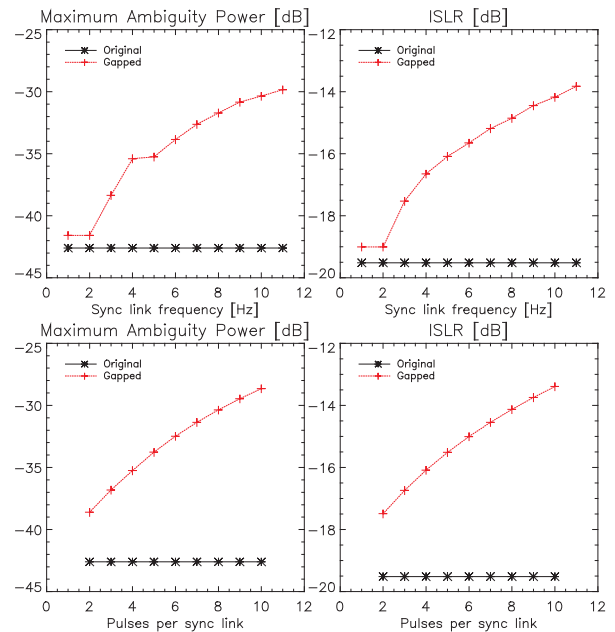

Figure 1. Variation of quality parameters, Maximum Ambiguity Power and ISLR, with increase in the synchronization frequency (top) and number of sync pulses (bottom).
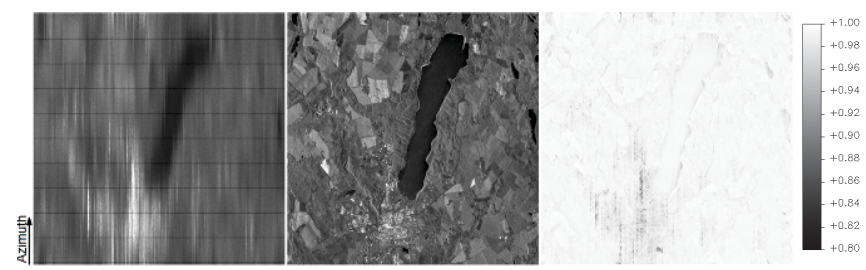

Figure 2. Gapped raw data (left); focused data with gaps in the raw data (middle); and coherence between images focused without gaps in the raw data and with gaps.

The variation of the ISLR and maximum ambiguity power with the increase in the synchronization link parameters (frequency $f \_s y n$ and number of exchange pulse per sync event $\left.n \_s y n\right)$ is shown in Figure 1. For the parameters of the order of the ones used in the TanDEM-X synchronization link $\left(n \_s y n \sim 4\right.$ and $f_{-} s y n \sim$ $5 \mathrm{~Hz}$ ), a noticeable degradation of the quality of the point target azimuthal IRF is observed (increase of around $7.7 \mathrm{db}$ in the maximum ambiguity power and of around $3.2 \mathrm{~dB}$ in the ISLR).

In real SAR images, a general increase in the noise level of the image will be observed as a result of the increased side lobes. This effect is more accentuated for urban areas, where the targets have a point-target like behavior with strong response and the increase in the side lobes energy is noticed by ghosts along azimuth. The effects might also be visible in areas with a very poor SNR, like flooded regions, due to the contribution of the increased side lobes from the surroundings. The smaller the oversampling rate of the system in azimuth and the higher the integration time (and consequently the higher the loss of information within the synthetic aperture), the more decreased the quality of the processed SAR product will be.

To illustrate the data corruption, the gaps introduced by the sync link of TanDEM-X bistatic operation were simulated in a stripmap TerraSAR-X acquisition. It was considered that 4 pulses are exchange per sync event and that the link has a frequency of $5 \mathrm{~Hz}$. Figure 2 shows the gapped raw data (left), the focused data with gaps (middle) and the coherence between the imaged focused without treating the gaps and the original focused image (right). The increase in the side lobes energy can be clearly seen by the presence of dark lines along azimuth in the coherence image in the region corresponding to an urban area (bottom).

\section{RECONSTRUCTION OF MISSING DATA}

As shown by the simulation results depicted in Figure 1, at least for point target-like targets, the loss in the image quality targets is relevant considering the TanDEM-X sync link operational parameters. Furthermore, due to the low oversampling rates usually used, the gaps introduced in the signal are larger than the allowed size given by the Nyquist rate preventing the use of loworder interpolators. Therefore more sophisticated reconstruction methods have to be considered.

As mentioned in Section 2, the loss of information in time domain has a corruptive effect spread through all spectral components in frequency domain. Assuming that the amount of data loss is sufficiently small and considering an appropriate estimator, the spectral content of the complete data can be estimated up to a certain precision from the spectral content of the available samples [6,7]. After this estimation, an inversion can be performed and the missing samples recovered. In this paper, three reconstruction methods based on spectral analysis are analyzed as possible solutions: The Papoulis-Gerchberg algorithm [5]; an autoregressive parametric estimator (AR-Burg) [1]; and a nonparametric estimation based on the Amplitude and Phase Estimation algorithm for gapped data (GAPES) [7,8]. For the application in TanDEM-X frame, in all the approaches the data is first range compressed and the estimation is performed considering one $1 \mathrm{D}$ azimuthal line at a time.

\subsection{Papoulis-Gerchberg (PG) estimation}

The Papoulis-Gerchberg (PG) method is a non-parametric interactive approach which uses a Fourier based low-pass filtering followed by a resample operation to estimate the spectrum. The method relies on the band limitation of the signal and on the fact that the missing samples will add high frequency components to the spectrum [5].

In each step, the signal is filtered according to its oversampling, and only the samples at the gaps positions are updated with the filtered values, operation known as resample. If the density of the signal (ratio between the number of available samples and the total number of samples) is greater or equal the bandwidth (ratio between number of null and non-null spectral components defined by the signal bandwidth), the convergence is assured [5]. Due to the TanDEM-X low oversampling rates, this criterion is often not satisfied, which might affect convergence. Furthermore, the envelope of the azimuth spectrum of the SAR raw data determined by the azimuthal antenna pattern contains side lobes, which goes against the band limitation hypotheses, also affecting convergence. Therefore, even when convergence can be reached, a large number of iterations might be necessary to obtain values of PSLR and maximum ambiguity power in the order of original ones [4], making the method unattractive for the operational use with TanDEM-X data.

\subsection{Auto-Regressive spectral estimation (AR-Burg)}

An alternative reconstruction can be performed using an autoregressive linear prediction model, as suggest in [1] for interpolating data resulting from interrupted SAR operation. In this case, the missing data is considered to be part of an auto-regressive stationary process. Once the coefficients modeling this process are 
estimated (e.g. using the Burg algorithm, which ensures stable models $[1,6])$, the missing samples can be reconstructed using an IIR filter. The reconstruction of the azimuthal signal is carried out locally in a gap to gap basis. For every gap, only a small segment of the available surrounding is considered for estimating the ARprocess coefficients. The choice of the segment size has to regard the order of the model (and ultimately the width of the gaps) and the hypothesis of stationarity. To reduce noise and improve the results, the estimation is carried out twice for each gap. Firstly, the segment previous to the gap is consider; secondly, the posterior one. The results are finally combined using cross-fading windows. This method is able to considerably compensate for the quality loss in urban areas using a single iteration and without oversampling requirements. However, for distributed areas, where the correlation between the missing data and the data in the surrounding is smaller, no relevant improvement might be expected.

\subsection{Amplitude and phase estimation of gapped data (GAPES)}

Another possibility is to use the amplitude and phase estimator for gapped data, proposed in $[7,8]$ for focusing SAR data with gapped phase history. It consists in a non-parametric adaptive filter-bank approach which uses minimum least squares criterion to estimate the spectrum and interactively invert the missing samples. In analogy to what is done for the AR-Burg approach, for the implementation in TanDEM-X frame, small segments around each gap in the azimuthal line are considered separately.

For the first iteration of the GAPES reconstruction, the spectral content of the missing data is assumed to be given by the available data. Hence, for each frequency of interest the spectral complex amplitude is estimated as the output of narrowband matched filter which satisfies the LS criterion using as input only the available samples $[7,8]$. The missing samples are then inverted in a way to minimize - in a least square sense - the error they introduce in the previously estimated spectrum. The spectrum is then iteratively reestimated considering all the samples and the oversample of the system (when available) and the missing samples are re-estimated.

The method is computationally heavier than the AR-Burg and PG algorithms. However, since only segments around the missing samples are considered, the convergence rate of the algorithm is not as much affected by the aperture size as the PapoulisGerchberg algorithm is (since the PG requires a filter applied on the whole synthetic aperture).

\section{EXPERIMENTAL RESULTS}

Figure 3 and 4 show coherence matrixes between the original focused image and the image focused after simulating the gaps of the TanDEM-X sync link and reconstructing the raw data. The sync link was simulated using $n \_s y n=4$ and $f \_s y n=5 \mathrm{~Hz}$. The region depicted in Figure 3 contains part of a lake (poor SNR), an urban area and a few agricultural fields. The region shown in Figure 4 is mainly composed by distributed targets. For the PG reconstruction 100 iterations were applied, while for the GAPES only 2 iterations. As can be noted by the reduction of the dark lines along azimuth in the coherence matrixes in Figure 3, the AR-Burg and GAPES estimators are able to considerably decrease the quality loss when applied to urban areas. Meanwhile the improvement with the PG algorithm is poorer. None of the algorithm is able to improve considerable the quality of the focused image over distributed areas. Note, however, that the general decrease in the coherence of distributed areas due to the missing data is fairly small (for example, the coherence of the image presented in Figure 4 focused without any gap treatment has a mean value of 0.99 and standard deviation of 0.007 ).

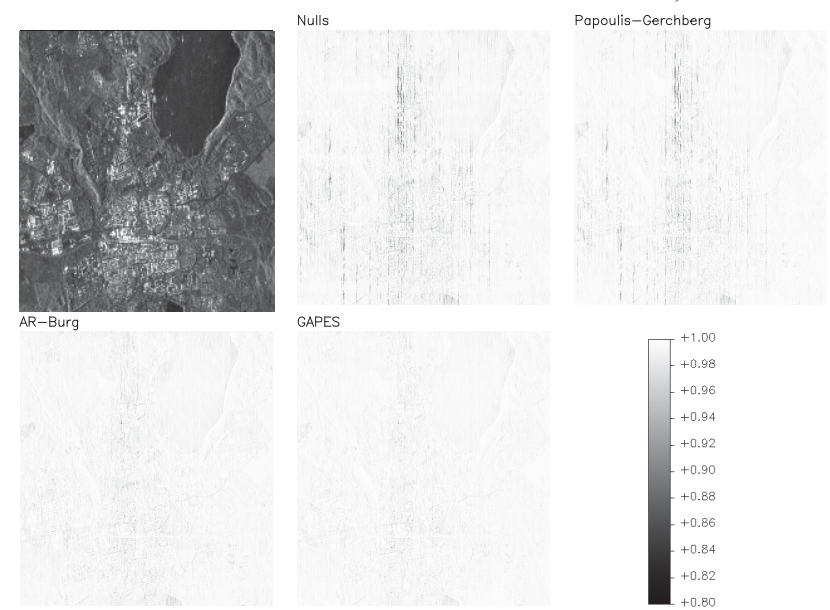

Figure 3. Coherence matrixes between the original focused image and reconstructed image using difference reconstruction schemes.

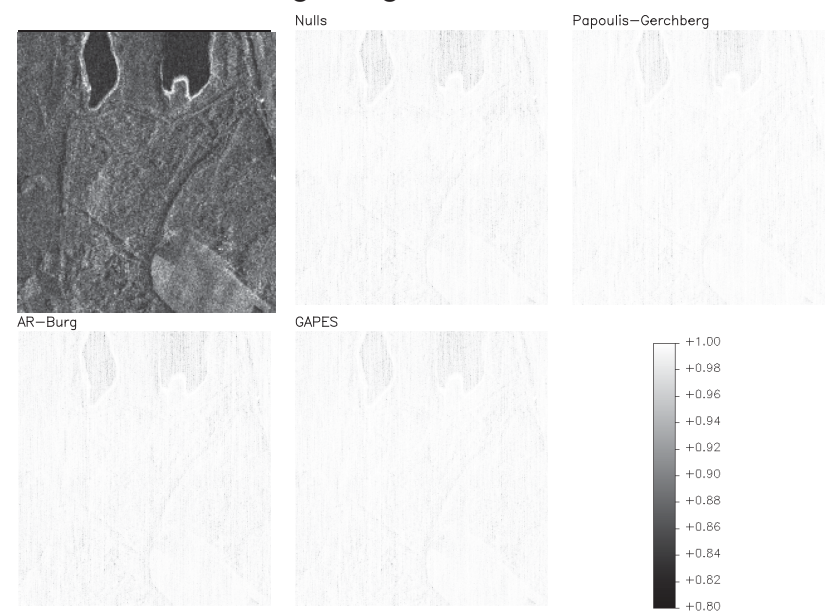

Figure 4. Coherence matrixes between the original focused image and reconstructed image using difference reconstruction schemes.

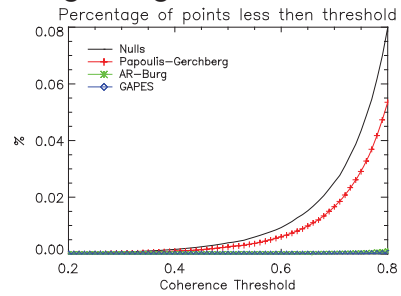

Figure 5. Cumulative histogram of region in Figure 3 considering coherence values between and 0.8 .

To allow a better visualization of the improvement for the urban areas, Figure 5 shows a cumulative histogram of the region presented in Figure 3, where the $x$-axis gives a coherence threshold and the $y$-axis gives the percentage of points in the image below this threshold (very small considering the total number of pixels in the block). Only coherence values below 0.8 are considered, which correspond mainly to the flooded areas and urban areas. 

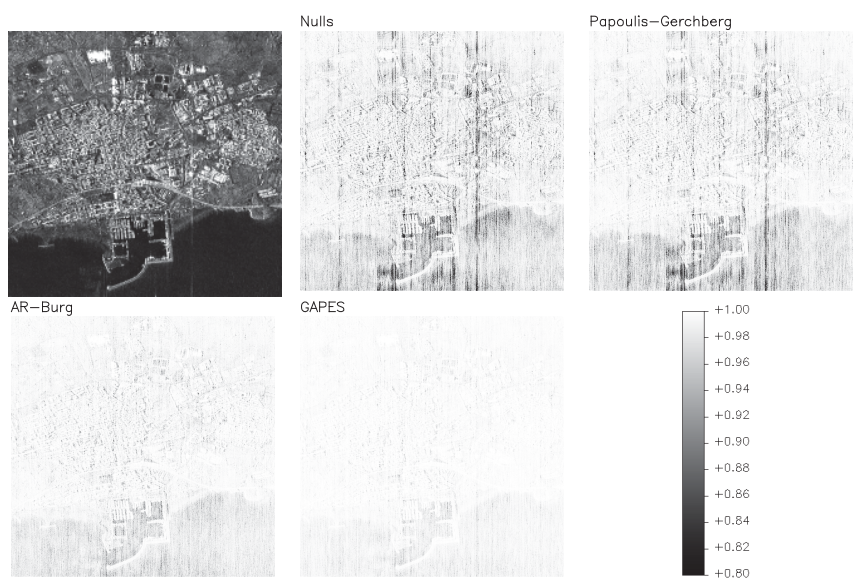

Figure 6. Coherence matrixes between the original focused image and reconstructed image using difference reconstruction schemes.

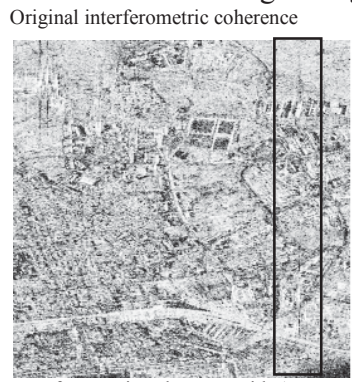
Interferometric coherence with Nulls

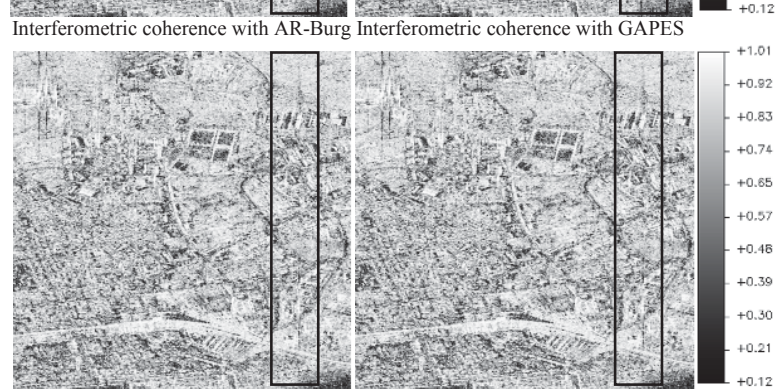

Figure 7. Zoom in the interferometric coherence using different reconstruction schemes for the corrupted master data.

The next results correspond to a TanDEM-X/TerraSAR-X pair acquired in the monostatic pursuit mode [2]. Since this mode does not require a synchronization link, the available original data can be used for validation. The gaps were once again simulated - for convenience only in the TanDEM-X data (master) - using the same sync link parameters as for the previous simulations. Figure 6 shows the resulting coherence matrixes between the original TanDEM-X image and the image focused after introducing the gaps and reconstructing the data. Finally, Figure 7 shows a zoom in the interferometric coherence. Although the effects in the interferometric coherence caused by the missing data are not so visible for the stripmap case, inside the marked box it is still possible to observe the disturbance in the amplitude of the master (darker line along azimuth) and the improvement achieved after the reconstruction. Figure 8 shows a zoom of the residual topographic phase. The effect of the missing data in the phase can be seen as the introduction of noise with zero mean. Overall, the interferometric phase is not much impacted, although the noise increase might be noticeable around targets with strong point target-like response.
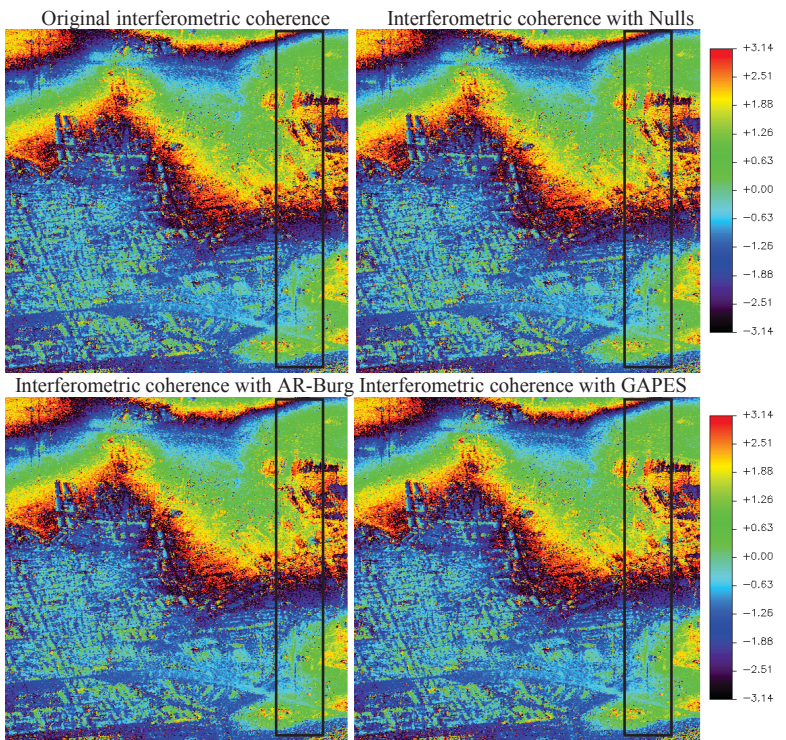

Figure 8. Zoom in the interferometric residual phase using different reconstruction schemes for the corrupted master data.

\section{CONCLUSIONS}

Different spectral estimation based approaches for reconstructing missing samples of SAR data for the case where the gaps are wider than the allowed by the Nyquist criterion were considered. Among the approaches, the AR-Burg and GAPES approach successful reconstruct point target- like data. However, their use should be restrained to urban areas, which are in fact the regions usually affected the most by the missing data.

\section{REFERENCES}

[1] Salzman, J.; Kirk, J. Interrupted Synthetic Aperture Radar, in IEEE AESS systems Magazine, 2002.

[2] Krieger, G. et al. TanDEM-X: A Satellite Formation for High-Resolution SAR Interferometry, in IEEE Transactions On Geoscience And Remote Sensing, vol. 45, 2007.

[3] Krieger, G.; Younis, M. Impact of Oscillator Noise in Bistatic and Multistatic SAR, in IEEE Geoscience and Remote Sense Letters, vol. 3, no. 3, 2006.

[4] Pinheiro, M; Rodriguez-Cassola, M. Reconstruction methods of missing SAR data: analysis in the frame of TanDEM-X synchronization link. In EUSAR 2012, vol. 1, 2012.

[5] Ferreira, P.J.S.G.; Digital Spectral Analysis with applications Interpolation and the discrete Papoulis-Garcheberg algortihm, in IEEE Transactions on Signal Processing, vol. 42, 1994.

[6] Marple, S.L.; Digital Spectral Analysis with applications, Prentice-Hall, 1987.

[7] Larsson, E.G.; Stoica, P.; Jian Li; Amplitude spectrum estimation for two-dimensional gapped data, in IEEE Transactions on Signal Processing,vol. 50, 2002.

[8] Larsson, E.G.; Stoica, P.; Jian Li; Matched-Filter bank interpretation of some spectral estimators, in Journal of Signal Processing, vol. 66, 1998. 